Nervenarzt 2021 · 92:571-578

https://doi.org/10.1007/s00115-021-01105-0

Angenommen: 23. Februar 2021

Online publiziert: 29. März 2021

(c) Springer Medizin Verlag GmbH, ein Teil von Springer Nature 2021

\author{
Kristina Adorjan $^{1,2} \cdot$ Robert Haussmann $^{3} \cdot$ Katrin Rauen $^{4,5} \cdot$ Oliver Pogarell $^{1}$ \\ 'LMU Klinikum, Klinik für Psychiatrie und Psychotherapie, Universität München, München, Deutschland \\ ${ }^{2}$ LMU Klinikum, Institut für Psychiatrische Phänomik und Genomik, Universität München, München, \\ Deutschland \\ ${ }^{3}$ Universitätsklinikum Carl Gustav Carus, Klinik und Poliklinik für Psychiatrie und Psychotherapie, \\ Universitäts Demenz Centrum (UDC), Technische Universität Dresden, Dresden, Deutschland \\ ${ }^{4}$ Klinik für Alterspsychiatrie, Psychiatrische Universitätsklinik Zürich, Zürich, Schweiz \\ ${ }^{5}$ LMU Klinikum, Institut für Schlaganfall- und Demenzforschung (ISD), Universität München, München, \\ Deutschland
}

\title{
Folgen der COVID-19-Pandemie für Menschen mit Schizophrenie, Demenz und Abhängigkeitserkrankungen
}

diction AND covid“ and „substance use disorders AND covid“.

\section{Folgen der COVID-19-Pandemie für Menschen mit einer Schizophrenie}

\section{Pandemieassoziierte psychotische Störungen}

Die virale Hypothese bezüglich der Entstehung der Schizophrenie ist seit Jahrzehnten bekannt [1]. Bereits nach der Spanischen-Grippe-Pandemie zu Beginn des 20. Jahrhunderts wurde eine Zunahme von Psychosen im späteren Lebensalter als Folge mütterlicher Infektionen während des 2. Trimesters der Schwangerschaft beobachtet. Erhöhte virale Antikörpertiter nach einer Virusinfektion durch Masern, Röteln, Varizellen, Zoster, Polio und Herpes bei Patienten mit Schizophrenie unterstützen diese Hypothese.

Severance et al. berichteten 2011 über den Zusammenhang zwischen einer Infektion durch Coronaviren und psychotischen Symptomen, indem sie die Immunoglobulin-G(IgG)-Reaktion gegen vier humane Coronavirusstämme bei Patienten mit psychotischer Erkrankung im Vergleich zu gesunden Kontrollen bestimmten und signifikant erhöhte IgG-Werte für zwei Coronavirusstämme (HKU1 und NL63) bei Patienten mit psychotischen Symptomen im Vergleich zu Kontrollprobanden zeigen konnten. Dies könnte darauf hindeuten, dass Coronaviren Risikofaktoren für neuropsychiatrische Erkrankungen sein könnten [2].

Die aktuelle Pandemie, bei der sich das SARS-CoV-2 (,severe acute respiratory syndrome coronavirus 2") weltweit ausbreitet, zeigt ähnliche Ergebnisse. Es gibt Hinweise auf neu auftretende Psychosen nach viraler Exposition, die möglicherweise auf eine neuronale Entzündung in relevanten Hirnregionen wie im präfrontalen, temporalen und parietalen Kortex zurückzuführen sind [3]. Fallberichten zufolge sind wahnhafte Symptome häufig inhaltlich mit COVID19 verbunden [4]. Zusätzliche Stressoren wie Isolation mit Kontaktbeschränkungen sowie Vereinsamung, Einkommensverluste und Arbeitslosigkeit können als weitere Umweltfaktoren maßgeblich an der Entwicklung psychotischer Störungen beteiligt sein [5]. Im Laufe der Zeit werden epidemiologische Daten zeigen, ob ähnlich wie nach der Influenzapandemie 1918/19 Schizophreniespektrumstörungen nach schweren Virusinfektionen zunehmen werden. 
Soziale Isolation als pandemieassoziierte Belastung

Die soziale Isolierung ist eine wichtige und unersetzliche Maßnahme zur Kontrolle der Ausbreitung der Pandemie und wirkt sich als zentraler Belastungsfaktor auf Menschen mit einer Schizophrenie aus. Auch die Aufrechterhaltung der psychiatrischen Versorgung in der COVID-19-Pandemie ist deutlich erschwert. Reduzierte Therapieprogramme, eingeschränkte Besuchsregeln und Belastungserprobungen, erkranktes Personal sowie Quarantäneverordnungen können psychiatrische Krankenhäuser daran hindern, ihre Patienten angemessen und ausreichend zu versorgen.

Für Patienten mit einer Schizophrenie können diese Maßnahmen fatale Folgen haben. Sie gelten als besonders gefährdet in der COVID-19-Pandemie. Menschen, die an einer Schizophrenie leiden, sind häufig von chronischen Krankheitsverläufen und einer reduzierten mittleren Lebenserwartung betroffen [6]. Ihr Zugang zu Wohn- und Bildungseinrichtungen und Aktivitäten sowie ihre sozialen Kontakte sind oft aufgrund ihrer mangelnden Kommunikationsfähigkeit und zwischenmenschlichen Fähigkeiten eingeschränkt. Durch ihre antipsychotische Medikation leiden sie häufig an einem metabolischen Syndrom, weshalb sie als Risikopatienten im Hinblick auf eine COVID-19-Erkrankung eingestuft werden. Eine weitere Herausforderung, die dringend der Aufmerksamkeit bedarf, sind die bisher unbekannten Auswirkungen der COVID-19-Erkrankung auf Menschen mit einer Schizophrenie hinsichtlich der Schwere der Symptome, der Rückfälle und der Notwendigkeit einer erhöhten Häufigkeit und Intensität der psychiatrischen Versorgung [7].

\section{》) Soziale Isolation erhöht insbesondere den Leidensdruck bei stationären Patienten}

Die Auswirkungen der sozialen Isolation bei Patienten mit Schizophrenie sind relevant und erhöhen insbesondere den Leidensdruck bei stationär behandelten Patienten mit einer Schizophrenie [8].
Bisherige Forschungsergebnisse konnten erhöhte Entzündungswerte (C-reaktives Protein), erhöhte Werte in der Hamilton Depression Scale (HAMD), der Hamilton Anxiety Scale (HAMA) und dem Pittsburgh Sleep Quality Index (PSQI) bei isolierten Patienten mit Schizophrenie im Vergleich zur nicht isolierten Kontrollgruppe zeigen. Dabei ist der PSQI ein gut etablierter Selbstbefragungsbogen zur Untersuchung der Schlafqualität und Unterscheidung von "guten“ und „schlechten“ Schläfern [9]. Unseres Wissens fehlen bisher Untersuchungen, die spezifische Assessments für Schizophrenie, wie z.B. die Positive and Negative Syndrome Scale (PANSS) oder Brief Psychiatric Rating Scale (BPRS), verwenden. Insgesamt führte eine soziale Isolation $\mathrm{zu}$ einer Verschlechterung der psychischen Symptomatik der Patienten. Vor diesem Hintergrund gelten psychiatrische Kliniken als wesentliche und unverzichtbare Elemente der medizinischen Versorgung. Daher ist es unerlässlich, die Funktionsfähigkeit psychiatrischer Krankenhäuser zu erhalten.

\section{Pandemieassoziierte Ein-} schränkungen in der Psychopharmakotherapie

Die Pandemie hat auch Auswirkungen auf die Psychopharmakotherapie der Schizophrenie. Die medikamentöse Behandlung einer Schizophrenie steht mit Veränderungen in drei Neurotransmittersystemen in Zusammenhang: dopaminerges, glutamaterges und serotonerges System. Bei den Antipsychotika der zweiten Generation („,atypische Neuroleptika“) handelt es sich um eine heterogene Gruppe von Medikamenten mit verschiedenem Rezeptorbindungsprofil. Sie wirken weniger im Bereich der $\mathrm{D}_{2}$ Rezeptoren-Blockade und verursachen deshalb weniger starke extrapyramidalmotorische Störungen. Sie entfalten ihre Wirkung vielmehr an den $\mathrm{D}_{1^{-}}$, $\mathrm{D}_{4}$ - und 5- $\mathrm{HT}_{2 \mathrm{~A}}$-Rezeptoren und reduzieren auch die Negativsymptomatik der Schizophrenie [10]. Die Blockade von $\mathrm{H}_{1}$-Rezeptoren sowie von $\alpha_{1-}$ und $\alpha_{2}$-Adrenorezeptoren erklären die unerwünschten Arzneimittelwirkungen dieser Medikamente: Gewichtszunah- me, metabolisches Syndrom, Sedierung, Akathisie und orthostatische Dysregulation [10].

\section{I) Verminderte psychiatrische Versorgung erschwert wichtige Routineuntersuchung}

Kontroversen sind in der psychiatrischen Therapie häufig, es besteht jedoch eine weitgehende Einigkeit über die besondere Rolle von Clozapin bei der Behandlung einer schweren refraktären Schizophrenie. Clozapin ist ein $\mathrm{D}_{2-}$ und 5- $\mathrm{HT}_{2 \mathrm{~A}}$-Rezeptor-Antagonist sowie ein 5 - $\mathrm{HT}_{1 \mathrm{~A}}$-Rezeptor-Agonist. Zudem bindet es an die $\alpha$-adrenergen sowie an muskarinische cholinerge Rezeptoren [10]. Nebenwirkungen sind Stoffwechselstörungen (Typ-2-Diabetes, Gewichtszunahme, Dyslipidämie) und kardiovaskuläre Störungen wie die Verlängerung des QT-Intervalls in der Elektrokardiographie sowie neurologische (Senkung der Anfallsschwelle) und hämatologische (Agranulozytose) Komplikationen. Gerade wegen des Risikos einer Clozapin-assoziierten schweren Agranulozytose ist eine regelmäßige Verlaufskontrolle die Voraussetzung für die Verabreichung von Clozapin auch während der Pandemie. Einschränkungen in der psychiatrischen Versorgung können jedoch den Zugang zu routinemäßigen Untersuchungen deutlich erschweren. Ein (abruptes) Absetzen von Clozapin birgt ein erhebliches Risiko für ein Rezidiv oder eine Verschlechterung des Schweregrads der Erkrankung und muss deshalb unbedingt vermieden werden [11].

Wenn während der Clozapin-Therapie Symptome einer grippalen Infektion wie Husten, Fieber, Schüttelfrost und Halsschmerzen auftreten, ist eine dringende ärztliche Beurteilung einschließlich eines vollständigen Blutbildes erforderlich. Es gibt nur begrenzte Informationen über die Auswirkungen des SARS-CoV-2-Virus auf die Neutropenie bei Personen, die Clozapin einnehmen [12], aber virale Erkrankungen können im Allgemeinen eine Neutropenie verursachen. Demnach könnte COVID-19 zusätzlich zu Clozapin zu einer Neu- 
tropenie führen. In diesem Fall kann die Clozapin-Dosis vorübergehend auf die Hälfte der ursprünglichen Dosis reduziert werden [11]. Ein komplettes Absetzen des Medikamentes muss im Regelfall nicht erfolgen. Im Falle einer bakteriellen Superinfektion sollte eine kalkulierte antibiotische Behandlung durchgeführt werden.

\section{Schlussfolgerung}

Zusammenfassend stellt die SARS-CoV2-Pandemie mit den langandauernden Kontaktbeschränkungen eine besondere Belastung für Menschen mit einer Schizophrenie dar und führt zu erhöhtem Leidensdruck durch limitierte psychiatrische, psycho- und sozialtherapeutische Behandlungsangebote. Ob eine Infektion mit SARS-CoV-2 ebenso, wie bereits für andere Coronaviren gezeigt, die Entstehung einer Schizophrenie begünstigt und/oder deren Exazerbation, ist Gegenstand der aktuellen Forschung. Bisher fehlen Untersuchungen, die spezifische Assessments für Schizophrenie, wie z. B. den PANSS oder BPRS, verwenden und sollten zukünftig zur Quantifizierung der Symptomschwere und -dauer in Zusammenhang mit der SARS-CoV-2Pandemie und COVID-19-Erkrankung bei Patienten mit einer Schizophrenie untersucht werden. Da virale Erkrankungen und im speziellen SARS-CoV-2 eine Neutropenie verursachen können, ist ein erhöhtes Monitoring bei der Verordnung von Psychopharmakotherapie mit einem erhöhten Agranulozytoserisiko, wie z. B. durch Clozapin, indiziert und stellt das psychiatrische Gesundheitssystem aufgrund der Kontaktbeschränkungen vor erhebliche Herausforderungen.

\section{Folgen der Covid-19-Pandemie für ältere Patienten mit kognitiven Störungen und Demenzerkrankungen}

Die COVID-19-Pandemie hat den Alltag vieler Menschen bereits erheblich verändert und zu einer Unterbrechung alltäglicher Routinen in allen Altersklassen geführt. Ihre drastischen Auswirkungen auf das soziale Leben sowie die direkten und indirekten Effekte einer SARS-CoV-

Nervenarzt 2021 -92:571-578 https://doi.org/10.1007/s00115-021-01105-0

(c) Springer Medizin Verlag GmbH, ein Teil von Springer Nature 2021

\section{K. Adorjan $\cdot$ R. Haussmann $\cdot$ K. Rauen $\cdot 0$. Pogarell}

\section{Folgen der COVID-19-Pandemie für Menschen mit Schizophrenie, Demenz und Abhängigkeitserkrankungen}

\section{Zusammenfassung}

Die COVID-19(",coronavirus disease 2019")Pandemie stellt die Sozial- und Gesundheitssysteme weltweit vor unerwartete Herausforderungen. Die direkten und indirekten medizinischen Folgen einer Infektion mit dem neuartigen Coronavirus bringen die Versorgungssysteme vielerorts an die Grenze ihrer Leistungsfähigkeit. Die neurotropen Effekte von COVID-19 können nicht nur neurologische, sondern auch psychische Akut- und Langzeitfolgen nach sich ziehen. Im psychiatrischen Kontext sind zusätzlich die psychischen und psychosozialen Folgen von Kontaktbeschränkungen und Lockdowns sowie die Auswirkungen täglicher medialer Berichterstattung auf Menschen mit psychischen Störungen zu berücksichtigen. In diesem Beitrag werden die Konsequenzen der COVID-19-Pandemie für Menschen mit psychischen Erkrankungen, insbesondere mit Schizophrenie, Demenz und Abhängigkeitserkrankungen, erörtert.

Schlüsselwörter

Pandemieassoziierte Belastung · Soziale Isolation · Akutfolgen · Langzeitfolgen . Psychische Erkrankungen

\section{Consequences of the COVID-19 pandemic for people with schizophrenia, dementia and substance use disorders}

\section{Abstract}

The coronavirus disease 2019 (COVID-19) pandemic poses unexpected challenges to social and healthcare systems worldwide. The direct and indirect medical consequences of infection with the novel coronavirus bring healthcare systems to their limits of their capabilities in many places. The neurotropic effects of COVID-19 can result not only in neurological but also in acute and long-term psychological sequelae. In the psychiatric context, the psychological and psychosocial consequences of contact restrictions and lockdowns as well as the effects of daily reports in the media on people with mental disorders must also be taken into consideration. In this article the consequences of the COVID-19 pandemic for people with mental illnesses, especially those with schizophrenia, dementia, and addictive diseases are discussed.

\section{Keywords}

Pandemic-associated burden · Social isolation · Acute sequelae $\cdot$ Long-term sequelae $\cdot$ Mental illness
2-Infektion beeinträchtigen jedoch insbesondere die psychische und körperliche Gesundheit hochvulnerabler, älterer Patienten mit kognitiven Störungen und Demenzerkrankungen [13].

\section{Infektionsrisiko und Folgen der sozialen Restriktionen}

Patienten mit Demenzerkrankungen haben einen begrenzten Zugang zu relevanten Informationen bezüglich der Pandemie sowie Schwierigkeiten, Maßnahmen zum Infektionsschutz zu erinnern und Quarantänemaßnamen adäquat umzusetzen, was das Infektionsrisiko dieser Patientengruppe deutlich erhöht [14].
Darüber hinaus können häufige Begleitsymptome wie Depressivität und Apathie eine geringere Adhärenz bezüglich der Hygieneregeln bedingen [15]. Insbesondere kognitive Beeinträchtigungen zeigten robuste Zusammenhänge mit einer COVID-19-Hospitalisierung [16].

\section{》) Soziale Deprivation \\ führt zu einer Zunahme neuropsychiatrischer Symptome}

Patienten mit Demenzerkrankungen haben häufig wenig soziale Bezüge und verfügen in der Regel über ein nur geringes Wissen hinsichtlich moder- 
ner Telekommunikationsmöglichkeiten, weshalb behördlich bestimmte Besuchsverbote in Pflegeheimen und Ausgangssperren beim älteren Menschen mit einer höheren Wahrscheinlichkeit zur sozialen Isolation führen [14]. Diese soziale Deprivation bedingt häufig eine Verschlechterung des kognitiven Funktionsniveaus und eine Neumanifestation oder Zunahme neuropsychiatrischer Symptome [13]. Neuropsychiatrische Symptome von Demenzpatienten während COVID-19-Pandemie-bedingter Restriktionen sind detailliert beschrieben worden [17]. Relevant sind hier Symptome wie Agitation, Ängstlichkeit, Apathie, Depressivität und Irritabilität $[18,19]$, wobei die Apathie das häufigste neuropsychiatrische Symptom darstellt [20].

Befragungen pflegender Angehörige von Patienten mit MCI („mild cognitive impairment") und Demenzerkrankungen ergaben, dass es während des Lockdowns zu einer signifikanten, allgemeinen Funktionsverschlechterung kam [21]. Insbesondere wurden Verschlechterungen in den Domänen Kommunikation, Bewegung, Stimmung und Kooperativität berichtet, was mit einer deutlichen Zunahme der Belastung der Angehörigen einherging [21]. Auch zunehmende Depressivität und Angstsymptome bei pflegenden Angehörigen während Lockdown und Quarantäne wurden beschrieben [22]. Die momentane Begrenzung ambulanter Hilfsangebote zur Entlastung der Angehörigen lässt die Versorgung von Patienten mit Demenzerkrankungen ebenfalls zu einer immensen Herausforderung werden.

\section{Mortalität und Folgen einer COVID-19-Erkrankung}

Patienten mit Demenzerkrankungen weisen zudem ein deutlich erhöhtes Risiko für schwere Erkrankungsverläufe einer SARS-CoV-2-Pneumonie auf. Bei etwa einem Sechstel der COVID-19assoziierten Todesfälle handelt es sich um Patienten mit Demenzerkrankungen [23]. Neben chronischen pulmonalen Erkrankungen zählen das Alter und die Alzheimer-Demenz zu den bedeutsamsten Mortalitätsfaktoren einer SARS-
CoV-2-Pneumonie [19, 23, 24]. Eine retrospektive Analyse einer italienischen Patientenkohorte zeigte beispielsweise eine deutlich höhere Sterblichkeit bei Patienten mit Demenz im Vergleich zu kognitiv gesunden Patienten (OR 1,84; $p<0,001$; [25]). Diesbezüglich muss jedoch auch das erhöhte Mortalitätsrisiko im Rahmen der häufigen Komorbiditäten arterielle Hypertonie, koronare Herzerkrankung und Diabetes mellitus berücksichtigt werden [24, 26]. Die beschriebenen Assoziationen zwischen dem Vorliegen eines ApolipoproteinE4(ApoE4)-Allels und einer deutlich erhöhten Mortalität im Rahmen einer COVID-19-Erkrankung lassen jedoch vermuten, dass die erhöhte Sterblichkeit nicht alleinig auf das Alter und die begleitenden Komorbiditäten zurückzuführen ist [27].

Darüber hinaus erleiden ältere Patienten häufiger neuropsychiatrische Komplikationen im Rahmen einer SARSCoV-2-Infektion. Eine Analyse von über 40.000 COVID-19-Patienten zeigte neuropsychiatrische Manifestationen bei 22,5\% der COVID-19-Patienten [28]. $\mathrm{Zu}$ nennen sind vor allem Insomnien, Depressionen, Kopfschmerzen und Enzephalopathien [28, 29]. Die potenziell irreversiblen kognitiven Defizite bei älteren Patienten nach durchgemachter COVID-19-Erkrankung sind annehmbar multifaktorieller Genese [29]. Als diesbezüglich relevante Faktoren sind langwierige Aufenthalte auf Intensivstationen mit invasiver Beatmung, sedierende Substanzen, eine systemische Inflammation, zerebrale Hypoxie sowie andere Organdysfunktionen und dyskognitive Effekte von Medikamenten zum Management der COVID-19Erkrankung zu nennen [29].

\section{》) Das hypoaktive Delir ist eine häufige Komplikation bei akuter SARS-CoV-2-Infektion}

Daneben sind auch direkte Effekte durch die neuroinvasiven Eigenschaften von SARS-CoV-2 sowie indirekte Effekte durch zerebrale Ischämien im Rahmen der SARS-CoV-2-induzierten endothelialen Dysfunktion zu berücksichtigen
[29]. Während der akuten SARS-CoV-2Infektion bei Patienten mit Demenz stellt zudem das hypoaktive Delir eine häufige Komplikation dar [25], welches in der Initialphase der Erkrankung klinisch nur schwer von einer Apathie abzugrenzen ist, die im Rahmen sozialer Deprivation ebenfalls häufig beobachtet wird. Diese häufig atypische Manifestation einer COVID-19-Erkrankung erschwert eine frühe Diagnosestellung und verzögert somit die Einleitung einer entsprechenden Therapie, was wiederum zu einer Verschlechterung der Prognose beitragen kann [29]. Neben dem gehäuften Auftreten hypoaktiver Delirien weisen Patienten mit Demenz und COVID19-Erkrankung seltener typische Leitsymptome wie Husten und Dyspnoe auf [23], was eine rechtzeitige Diagnosestellung zusätzlich erschwert und auch das Ergreifen von Infektionsschutzmaßnahmen verzögert.

\section{Perspektive}

Sowohl die präventiven Maßnahmen zur Eindämmung der SARS-CoV-2Pandemie als auch die unmittelbaren Folgen einer Infektion treffen Patienten mit Demenzerkrankungen besonders hart. Die Schließungen von Tagespflegeeinrichtungen sowie mehrmonatige Besuchsverbote in Pflegeheimen haben $\mathrm{zu}$ drastischen Einschränkungen in der Versorgung von Patienten mit Demenz und auch zu einer erheblichen Reduktion von Unterstützungsangeboten für pflegende Angehörige geführt [30]. Der Einsatz telekommunikativer Strategien ist bei diesem Patientenklientel meist in nur sehr begrenztem Umfang möglich. Auch hinsichtlich der medizinischen Versorgung ist damit zu rechnen, dass das gegenwärtige Umlenken von Ressourcen im Gesundheitssystem annehmbar wahrscheinlich $\mathrm{zu}$ einer Verschlechterung der medizinischen Versorgung von Patienten mit Demenz führen wird $[15,29]$. Insbesondere die ambulante Demenzdiagnostik unterliegt in einer Pandemie, im Vergleich zu anderen Bereichen, disproportionalen Restriktionen, denn das aktuelle Infektionsrisiko im Rahmen wiederholter ambulanter Termine für Labordiagnostik, zerebrale 
Bildgebung und differenzierende Testpsychometrie übersteigt möglicherweise den klinischen Nutzen einer frühen Demenzdiagnose und eines zeitgerechten klinischen Monitorings [15].

Im bisherigen Verlauf der Pandemie waren die meisten COVID-19-assoziierten Todesfälle bei Patienten aus Pflegeeinrichtungen $\mathrm{zu}$ verzeichnen, in denen zu einem großen Anteil Patienten mit Demenzerkrankungen leben. Dies verdeutlicht die unbedingt notwendige Intensivierung von Präventionsmaßnahmen und die konsequente Umsetzung von Hygienekonzepten zum Schutz dieses vulnerablen Patientenklientels [29]. Es bedarf hier jedoch einer wohlüberlegten Abwägung zwischen der unbedingt notwendigen Prävention von Infektionen einerseits und der Zumutbarkeit der veranlassten Maßnahmen auf der anderen Seite.

Auch die Therapie einer COVID19-Erkrankung bei Patienten mit Demenzerkrankungen stellt, in Anbetracht der hohen Mortalität und auch vor dem Hintergrund der häufig irreversiblen kognitiven Befundverschlechterung im Rahmen einer SARS-CoV-2-Pneumonie, eine immense klinische und medizinethische Herausforderung für die Behandler dar. Die aktuell medial häufig lebhaft diskutierte Frage der Triage von COVID-19-Patienten muss in Anbetracht steigender Fallzahlen und begrenzter Intensivbehandlungskapazitäten diskutiert werden. Patientenverfügungen und Vorsorgevollmachten können ärztlichen Behandlern insbesondere in dieser Situation als wichtige Entscheidungshilfen dienen. Es bleibt zu hoffen, dass die nun initiierten Schutzimpfungen $\mathrm{zu}$ einer baldigen Entlastung des Gesundheitswesens und darüber hinaus zu einer Verbesserung der Versorgung von Patienten mit Demenzerkrankungen führen. Doch bis dahin bleibt die Pandemie eine große Herausforderung und Belastung für Patienten mit Demenzerkrankungen, pflegende Angehörige und Behandler.

Folgen der COVID-19-

\section{Pandemie für Menschen mit Abhängigkeitserkrankungen}

Menschen mit Suchterkrankungen sind in mehrfacher Hinsicht von der Pandemie beeinträchtigt. Bei den Betroffenen handelt es sich um eine oftmals marginalisierte und stigmatisierte Patientengruppe, die einen hohen Betreuungsbedarf aufweist, aber bereits unter regulären Bedingungen teils nur begrenzt, einen dem Störungsbild angemessenen Zugang zum medizinischen Hilfesystem erhält bzw. Hilfe in Anspruch nehmen kann [31]. Zudem gehören Menschen mit Suchterkrankungen aufgrund der hohen Krankheitslast durch Komorbiditäten einer Risikogruppe mit erhöhtem Infektionsrisiko und ungünstiger Prognose im Falle einer COVID-19-Erkrankung an [32].

\section{Alkohol, Tabak, Cannabis}

Psychosoziale Stressoren, ausgelöst und/ oder verstärkt durch längere soziale Isolierung infolge von Kontaktbeschränkungen, stellen Risikofaktoren für erhöhten Substanzkonsum dar und können bei Menschen mit Abhängigkeitserkrankungen zu erhöhtem Craving mit Rückfallrisiko führen. Umgekehrt sind Konsumsteigerungen selbst ebenfalls mit negativen Auswirkungen auf physiologische Stressreaktionen verbunden, woraus wechselseitig negative Konsequenzen resultieren [33]. Erste Untersuchungen der Lockdownfolgen auf den Suchtmittelkonsum (Alkohol, Tabak, Cannabinoide) in Deutschland und Belgien deuten auf eine moderate Zunahme des Substanzkonsums im Rahmen von Kontaktbeschränkungen und Quarantänemaßnahmen hin [34, 35].

\section{》) Erhebungen weisen auf eine leichte Konsumzunahme von Alkohol und Tabak hin}

In einer anonymen Onlinebefragung während der Kontaktbeschränkungen im Frühjahr 2020 gaben von über 2000 Teilnehmern über ein Drittel der Befragten an, seit Lockdownbeginn mehr zu trin- ken, knapp $20 \%$ tranken weniger und $8 \%$ tranken keinen Alkohol [34]. In einer vergleichbaren Web-basierten Erhebung in Belgien an über 3600 Personen zeigten Vanderbruggen et al. eine geringe, aber signifikante Zunahme des Alkohol- und Zigarettenkonsums, wohingegen der Cannabiskonsum sich nicht signifikant veränderte. Die berichteten Motive des vermehrten Konsums waren Langeweile, Mangel an Sozialkontakten und Verlust der Tagesstruktur sowie Einsamkeit [35]. Neben diesen empirischen Befunden, die trotz methodischer Limitationen auf eine leichte Konsumzunahme in der Bevölkerung hinweisen, sind aus epidemiologischer Sicht grundsätzlich zwei Szenarien in Betracht zu ziehen [36]:

- einerseits kann die Zunahme psychosozialer Stressoren in Kombination mit sozialer Isolierung und finanziellen Schwierigkeiten sowie Zukunftsunsicherheit gerade im Hinblick auf Alkohol zu einer Konsumzunahme führen,

- andererseits können finanzielle Einschränkungen, Kontaktbeschränkungen und Trinkverbote in der Öffentlichkeit auch gegenteilige Auswirkungen haben [36].

\section{Illegale Drogen}

Die Auswirkung von Kontaktbeschränkungen und „social distancing“ auf den Konsum illegaler Drogen wurde von der Europäischen Monitoringstelle für Drogen und Drogenabhängigkeit (EMCDDA) im Juni 2020 untersucht. Insgesamt zeigte sich während der ersten Monate der Pandemie im Frühjahr 2020 eine Abnahme des Drogenkonsums, der mutmaßlich auf Kontakt- und Ausgangsbeschränkungen mit entsprechenden Auswirkungen auf die Drogenszene und eine Abnahme der Verfügbarkeit verschiedener Substanzen zurückzuführen war [37].

\section{》) Gerade im Lockdown ist der Zugang zu ausdifferenzierten Therapien entscheidend}

Kontaktbeschränkungen und Angebotsverknappungen betrafen vor allem 
Tab. 1 Substanzkonsum und Risikofaktoren für bzw. bei COVID-19. (Mod. nach [35])

\begin{tabular}{|c|c|c|c|c|c|}
\hline Substanz & Immunsystem & $\begin{array}{l}\text { Respiratorisches } \\
\text { System }\end{array}$ & $\begin{array}{l}\text { Kardiovaskuläres } \\
\text { System }\end{array}$ & ZNS & COVID-19 \\
\hline Nikotin & $\begin{array}{l}\text { Inflammatorische Zytokine } \uparrow \\
\text { Antiinflammatorische Faktoren } \downarrow \\
\text { Immunsuppression }\end{array}$ & COPD (Tabak) & $\begin{array}{l}\text { Kardiovaskuläre Ri- } \\
\text { sikofaktoren (Tabak) }\end{array}$ & $\begin{array}{l}\text { Störung der } \\
\text { Blut-Hirn-Schranke }\end{array}$ & \multirow{5}{*}{$\begin{array}{l}\text { Infektionsrate } \uparrow \\
\text { Gestörte Immunant- } \\
\text { wort } \\
\text { ZNS-Beteiligung } \uparrow \\
\text { Schwere der } \\
\text { Erkrankung } \uparrow \\
\text { Opportunistische } \\
\text { Infektionen } \uparrow \\
\text { Mortalität } \uparrow\end{array}$} \\
\hline $\mathrm{THC} / \mathrm{CBD}$ & $\begin{array}{l}\text { Immunsuppression, verminder- } \\
\text { te Antikörperresponse }\end{array}$ & $\begin{array}{l}\text { COPD (bei Kombi- } \\
\text { nation mit Tabak) }\end{array}$ & $\begin{array}{l}\text { Hypertonie, Tachykar- } \\
\text { die }\end{array}$ & $\begin{array}{l}\text { Störung der } \\
\text { Blut-Hirn-Schranken (bei } \\
\text { Kombination mit Tabak) }\end{array}$ & \\
\hline Alkohol & $\begin{array}{l}\text { Proinflammatorische Zytokine } \uparrow \\
\text { Hyposplenismus }\end{array}$ & $\begin{array}{l}\text { Erhöhtes Pneumo- } \\
\text { nierisiko }\end{array}$ & $\begin{array}{l}\text { Kardiomyopathie, Hy- } \\
\text { pertonie }\end{array}$ & \multirow[t]{2}{*}{$\begin{array}{l}\text { Erhöhte Permeabilität der } \\
\text { Blut-Hirn-Schranke }\end{array}$} & \\
\hline Opioide & $\begin{array}{l}\text { Neuroinflammatorisch, gestörte } \\
\text { HPA-Achse, verminderte Gluko- } \\
\text { kortikoidausschüttung }\end{array}$ & Atemdepression & $\begin{array}{l}\text { Orthostatische Dysre- } \\
\text { gulation, Dysrhythmien }\end{array}$ & & \\
\hline $\begin{array}{l}\text { Kokain und } \\
\text { Stimulanzien }\end{array}$ & $\begin{array}{l}\text { HPA-Achsen-Aktivierung, Im- } \\
\text { munsuppression, neuroinflam- } \\
\text { matorisch }\end{array}$ & $\begin{array}{l}\text { Pneumopathie, } \\
\text { Dyspnoe }\end{array}$ & $\begin{array}{l}\text { Arrhythmien, Hyperto- } \\
\text { nie, Kardiomyopathie, } \\
\text { Thromboserisiko, pul- } \\
\text { monaler Hypertonus, } \\
\text { zerebrale Ischämie und } \\
\text { Hypoxie }\end{array}$ & $\begin{array}{l}\text { Störung der } \\
\text { Blut-Hirn-Schranke, er- } \\
\text { höhte Permeabilität }\end{array}$ & \\
\hline
\end{tabular}

Hochrisikoopioidkonsumenten („high risk opioid users“), was einerseits zu riskanteren Konsumformen (synthetische Opioide wie Fentanyl, neue psychoaktive Substanzen) führen konnte oder zu einer zunehmenden Nachfrage nach einer niedrigschwelligen Substitutionsbehandlung. Letzteres wurde u.a. in Deutschland berichtet, auch dies wiederum möglicherweise als Folge einer reduzierten Verfügbarkeit illegalen Heroins auf dem Schwarzmarkt oder geringerer finanzieller Möglichkeiten. Gerade im Rahmen einer Pandemie mit Lockdown, Kontaktbeschränkungen sowie gravierenden wirtschaftlichen Folgen für die Betroffenen ist somit die Aufrechterhaltung ausdifferenzierter Therapieoptionen entscheidend [38, 39].

Neben allgemeinen sozialen, sozioökonomischen und medizinischen Auswirkungen sind zusätzlich die spezifischen Gesundheitskrisen der Konsumenten legaler oder illegaler Drogen im Hinblick auf die Folgen einer Infektion mit SARS-CoV-2 zu berücksichtigen. Patienten mit Abhängigkeitserkrankungen haben nicht nur aufgrund von Stigmatisierung und Diskriminierung begrenzten Zugang zu einer umfassenden Gesundheitsversorgung, sondern sind zusätzlich durch psychische und somatische Komorbidität belastet, die direkt oder indirekt die Suszeptibilität für Infektionserkrankungen erhöhen und die Prognose insgesamt verschlechtern [40].

\section{Risiken für Menschen mit Abhängigkeitserkrankungen}

Eine gravierende gesundheitliche Pandemiefolge für Menschen mit Suchterkrankungen stellt die erhöhte Infektanfälligkeit dar. Diese kann sich aus suchtassoziierten Verhaltensmustern ergeben, die wiederum durch die oben geschilderten psychosozialen Folgen der Pandemie verstärkt werden können. Menschen mit Suchterkrankungen erleben im Rahmen von Craving Kontrollverlust und enthemmtes Verhalten, das mit geringerem Selbstschutz bzw. Gesundheitsvorsorge und daraus bedingtem erhöhtem Ansteckungsrisiko verbunden sein kann [38, 41, 42]. Darüber hinaus weisen Menschen mit Abhängigkeitserkrankungen ein hohes Komorbiditätsrisiko auf, wie z.B. Herz-Kreislauf-Erkrankungen, chronische Lungenerkrankungen und andere Infektionserkrankungen, die den Verlauf und Prognose einer COVID19-Erkrankung ungünstig beeinflussen können [5, 43].

Neben den komorbiden somatischen Erkrankungen können Substanzkonsumstörungen auch $\mathrm{zu}$ einer Beeinträchtigung des Immunsystems führen,
z.B. durch Beeinflussung proinflammatorischer Zytokine (Alkohol, Nikotin), immunmodulatorischer bzw. immunsuppressiver Effekte (Cannabidiol, Tetrahydrocannabinol) oder Beeinflussung der HPA(Hypothalamus-HypophysenNebennierenrinden)-Stressachse (Opioide, Stimulanzien). Im Hinblick auf eine Infektion mit SARS-CoV-2 und der Entwicklung von COVID-19 ist ebenfalls hervorzuheben, dass viele psychotrope Substanzen die Blut-Hirn-SchrankenPermeabilität beeinflussen und dadurch möglicherweise die neurotropen Effekte von SARS-CoV-2 und somit die Prognose einer COVID-19-Erkrankung ungünstig beeinflussen ([44]; • Tab. 1).

\section{Opioidabhängigkeit und Substitutionstherapie}

Die Substitutionsbehandlung ist weltweit anerkannt die Therapie der Wahl bei Menschen mit Opioidabhängigkeit, setzt aber dauerhaft verlässliche Rahmenbedingungen voraus $[39,45]$. Bei der oben ausgeführten vulnerablen Gruppe der Personen mit Opioidabhängigkeit in Substitutionstherapie stellt das Vermeiden pandemieassoziierter Therapieabbrüche eine zentrale Herausforderung dar. Dies wurde in Deutschland von Institutionen, Behörden und Kostenträgern frühzeitig erkannt und in der SARS-CoV-2-Arz- 
neimittelversorgungsverordnung vom 20.04.2020 berücksichtigt. Auf dieser gesetzlichen Grundlage wurden strukturelle und konzeputelle Anpassungen der Substitutionsbehandlung an Folgen und Auswirkungen der Pandemie ermöglicht [39].

\section{Telemedizin}

Telemedizinische Angebote erleichtern die Betreuung der Patienten außerhalb regulärer Zeiten und ermöglichen einen sicheren Zugang zu Beratungsangeboten. Während der Lockdownphasen wurden vielerorts telemedizinische Angebote diskutiert und technische Voraussetzungen hierfür geschaffen. Hierdurch ließen sich Kontakte reduzieren und zumindest die psychosoziale Betreuung der Betroffenen sicherstellen. Auch mit diesem Ansatz konnten stabil auf ein Substitut eingestellte Personen bei Ausweitung der Take-home-Vergabe adäquat betreut werden. Dennoch ist eine Vielzahl technologischer Herausforderungen und Hürden zu berücksichtigen. Gerade in der Substitutionstherapie erfordern Sorgfaltspflicht, Aufrechterhaltung von Qualität und Sicherheit und nicht zuletzt die gesetzlichen Vorgaben (Betäubungsmittel-Verschreibungsverordnung) nach wie vor auch persönliche Kontakte [46].

\section{Fazit für die Praxis}

- Die psychosozialen Auswirkungen der COVID-19(",coronavirus disease 2019“)-Pandemie betreffen vor allem die Gruppe hochvulnerabler Patienten mit psychiatrischen Erkrankungen wie beispielsweise Schizophrenie, kognitiven Störungen und Demenzerkrankungen sowie Abhängigkeitserkrankungen.

- Die Pandemie hat nicht nur Auswirkungen auf die Versorgungssituation von Menschen mit einer Schizophrenie, sondern auch auf ihre Psychopharmakotherapie (z. B. Clozapin).

- Diese vulnerable Patientenkohorte weist ein erhöhtes Infektionsrisiko und auch ein erhöhtes Risiko für schwere Erkrankungsverläufe auf.
- Häufig treten bei Patienten mit kognitiven Störungen neuropsychiatrische Komplikationen wie Insomnie, Depression, Kopfschmerzen und zunehmende kognitive Defizite auf.

- Insbesondere das Alter und die Demenz vom Alzheimer-Typ sind bedeutsame Mortalitätsfaktoren einer SARS-CoV-2(„,severe acute respiratory syndrome coronavirus $2^{\prime \prime}$ )Infektion.

- Patienten mit Abhängigkeitserkrankungen sind häufig durch somatische Komorbiditäten belastet, die direkt oder indirekt die Suszeptibilität für Infektionserkrankungen erhöhen und die Prognose insgesamt verschlechtern.

\section{Korrespondenzadresse}

\section{PD Dr. Kristina Adorjan}

LMU Klinikum, Klinik für Psychiatrie und Psychotherapie, Universität München Nussbaumstr. 7, 80336 München, Deutschland kristina.adorjan@

med.uni-muenchen.de

Danksagung. egePan Unimed wird im Rahmen des Netzwerks Universitätsmedizin (NUM) vom Bundesministerium für Bildung und Forschung (BMBF) gefördert (Förderkennzeichen: 01KX2021) und geleitet von Prof. Dr. Jochen Schmitt und Dr. Michael von Wagner.

\section{Einhaltung ethischer Richtlinien}

Interessenkonflikt. K. Adorjan, R. Haussmann, K. Rauen und O. Pogarell geben an, dass kein Interessenkonflikt besteht.

Für diesen Beitrag wurden von den Autoren keine Studien an Menschen oder Tieren durchgeführt. Für die aufgeführten Studien gelten die jeweils dort angegebenen ethischen Richtlinien.

\section{Literatur}

1. Kępińska AP, lyegbe $C O$, Vernon $A C$, Yolken $R$, Murray RM, Pollak TA (2020) Schizophrenia and influenza at the centenary of the 1918-1919 Spanish influenza pandemic: mechanisms of psychosis risk. Front Psychiatry 11:72. https://doi. org/10.3389/fpsyt.2020.00072

2. Severance EG, Dickerson FB, Viscidi RP, Bossis I, Stallings CR, Origoni AE, Sullens A, Yolken RH (2011) Coronavirus immunoreactivity in individuals with a recent onset of psychotic symptoms. Schizophr Bull 37(1):101-107. https://doi.org/10.1093/ schbul/sbp052

3. Zimmer A, Youngblood A, Adnane A, Miller BJ, Goldsmith DR (2021) Prenatal exposure to viral infection and neuropsychiatric disorders in offspring: a review of the literature and recommendations for the COVID-19 pandemic Brain Behav Immun 91:756-770

4. Smith CM, Komisar JR, Mourad A, Kincaid BR (2020) COVID-19-associated brief psychotic disorder. BMJ Case Rep 13(8):e236940. https://doi.org/10.1136/ bcr-2020-236940

5. Dunlop A, Lokuge B, Masters $D$, Sequeira $M$, Saul P, Dunlop G, Ryan J, Hall M, Ezard N, Haber P, Lintzeris N, Maher L (2020) Challenges in maintaining treatment services for people who use drugs during the COVID-19 pandemic. Harm Reduct J 17(1):26. https://doi.org/10.1186/ s12954-020-00370-7

6. Charlson FJ et al (2018) Global epidemiology and burden of schizophrenia: findings from the global burden of disease study 2016. Schizophr Bull 44(6):1195-1203

7. Kaufman KR, Petkova E, Bhui KS, Schulze TG (2020) A global needs assessment in times of a global crisis: world psychiatry response to the COVID-19 pandemic. BJPsych Open 6(3):e48. https://doi.org/ 10.1192/bjo.2020.25

8. Ma J, Hua T, Zeng K, Zhong B, Wang G, Liu X (2020) Influence of social isolation caused by coronavirus disease 2019 (COVID-19) on the psychological characteristics of hospitalized schizophrenia patients: a case-control study. Transl Psychiatry 10(1):411. https://doi.org/10.1038/s41398-02001098-5

9. Buysse DJ, Reynolds CF 3rd, Monk TH, Berman SR, Kupfer DJ (1989) The Pittsburgh Sleep Quality Index: a new instrument for psychiatric practice and research. Psychiatry Res 28(2):193-213. https://doi.org/10.1016/0165-1781(89)90047-4

10. Dreher J (Hrsg) (2019) Psychopharmakotherapie griffbereit. Medikamente, psychoaktive Genussmittel und Drogen, 4. Aufl. Thieme, Stuttgart, S62-109

11. Siskind D, Honer WG, Clark S, Correll CU, Hasan A, Howes O, Kane JM, Kelly DL, Laitman R, Lee J, MacCabe JH, Myles N, Nielsen J, Schulte PF, Taylor D Verdoux H, Wheeler A, Freudenreich $O$ (2020) Consensus statement on the use of clozapine during the COVID-19 pandemic. J Psychiatry Neurosci 45(3):222-223

12. Dragoi AM, Radulescu I, Năsui BA, Pop AL, Varlas VN, Trifu S (2020) Clozapine: an updated overview of pharmacogenetic biomarkers, risks, and safety-particularities in the context of COVID19. Brain Sci 10(11):840. https://doi.org/10.3390/ brainsci10110840

13. Manca R, De Marco M, Venneri A (2020) The impact of COVID-19 infection and enforced prolonged social isolation on neuropsychiatric symptoms in older adults with and without dementia: a review. Front Psychiatry 11:585540

14. Wang H, Li T, Barbarino Pet al (2020) Dementia care during COVID-19. Lancet 395:1190-1191

15. BrownEE, Kumar S, Rajji TKetal (2020) Anticipating and mitigating the impact of the COVID-19 pandemic on Alzheimer's disease and related dementias. Am J Geriatr Psychiatry 28:712-721

16. Batty GD, Deary IJ, Luciano M et al (2020) Psychosocial factors and hospitalisations for COVID-19: prospective cohort study based on a community sample. Brain Behav Immun 89:569-578

17. Simonetti A, Pais C, Jones M et al (2020) Neuropsychiatric symptoms in elderly with dementia during COVID-19 pandemic: definition, treatment, and future directions. Front Psychiatry 11:579842 
18. Canevelli M, Valletta $M$, Toccaceli Blasi $M$ et al (2020a) Facing dementia during the COVID-19 outbreak. J Am Geriatr Soc 68:1673-1676

19. Covino M, De Matteis G, Santoro M et al (2020) Clinical characteristics and prognostic factors in COVID-19 patients aged $\rangle /=80$ years. Geriatr Gerontol Int 20:704-708

20. Canevelli M, Bruno G, Cesari M (2020b) Providing simultaneous COVID-19-sensitive and dementiasensitive care as we transition from crisis care to ongoing care. J Am Med Dir Assoc 21:968-969

21. Tsapanou A, Papatriantafyllou JD, Yiannopoulou K, Sali D, Kalligerou F, Ntanasi E, Zoi P, Margioti E, Kamtsadeli V, Hatzopoulou M, Koustimpi M, Zagka A, Papageorgiou SG, Sakka P (2021) The impact of COVID-19 pandemic on people with mild cognitive impairment/dementia and on their caregivers. Int J Geriatr Psychiatry. 36(4):583-587. https://doi.org/ 10.1002/gps.5457

22. Altieri M, Santangelo G (2021) The psychological impact of COVID-19 pandemic and Lockdown on Caregivers of people with dementia. Am J Geriatr Psychiatry 29:27-34

23. Canevelli M, Palmieri L, Raparelli V et al (2020c) Prevalence and clinical correlates of dementia among COVID-19-related deaths in Italy. Alzheimers Dement 12:e12114

24. Wu JT, Leung K, Bushman Met al (2020) Estimating clinical severity of COVID-19 from the transmission dynamics in Wuhan, China. Nat Med 26:506-510

25. Bianchetti A, Rozzini R, Guerini Fetal (2020) Clinica presentation of COVID19 in dementia patients. J Nutr Health Aging 24:560-562

26. Bauer K, Schwarzkopf L, Graessel E et al (2014) A claims data-based comparison of comorbidity in individuals with and without dementia. BMC Geriatr 14:10

27. Kuo CL, Pilling LC, Atkins JL et al (2020) ApoE e4e4 genotype and mortality with COVID-19 in UK Biobank. J Gerontol A Biol Sci Med Sci 75:1801-1803

28. Nalleballe K, Reddy Onteddu S, Sharma R et al (2020) Spectrum of neuropsychiatric manifestations in COVID-19. Brain Behav Immun 88:71-74

29. Alonso-Lana S, Marquie M, Ruiz A et al (2020) Cognitive and neuropsychiatric manifestations of COVID-19 and effects on elderly individuals with dementia. Front Aging Neurosci 12:588872

30. Barros D, Borges-Machado F, Ribeiro O et al (2020) Dementia and COVID-19: The Ones Not to Be Forgotten. Am J Alzheimers Dis Other Demen 35:1533317520947505

31. Kraus L, Piontek D, Pfeiffer-Gerschel T, Rehm J (2015) Inanspruchnahme gesundheitlicher Versorgung durch Alkoholabhängige. Suchttherapie 16(1):18-26

32. Volkow ND (2020) Collision of the COVID19 and Addiction Epidemics. Ann Intern Med 173(1):61-62. https://doi.org/10.7326/M20-1212

33. Koob GF, Powell P, White A (2020) Addiction as a coping response: hyperkatifeia, deaths of despair, and COVID-19. Am J Psychiatry 177(11):1031-1037. https://doi.org/10.1176/ appi.ajp.2020.20091375

34. Koopmann A, Georgiadou E, Kiefer F, Hillemacher T (2020) Did the general population in Germany drink more alcohol during the COVID-19 pandemic lockdown? Alcohol Alcohol 55(6):698-699.https:// doi.org/10.1093/alcalc/agaa058

35. Vanderbruggen $\mathrm{N}$, Matthys $\mathrm{F}$, Van Laere $\mathrm{S}$ Zeeuws D, Santermans L, Van den Ameele $S$, Crunelle CL (2020) Self-reported alcohol, tobacco, and cannabis use during COVID-19 Lockdown measures: results from a web-based survey. Eur
Addict Res 26(6):309-315. https://doi.org/10 1159/000510822

36. Rehm J, Kilian C, Ferreira-Borges $C$, Jernigan $D$ Monteiro M, Parry CDH, Sanchez ZM, Manthey J (2020) Alcohol use in times of the COVID 19: Implications for monitoring and policy. Drug Alcohol Rev 39(4):301-304. https://doi.org/10. 1111/dar.13074

37. EMCDDA EMCDDA Trendspotter briefing: impact of COVID-19 on patterns of drug use and drug-related harms in Europe, June 2020. https://www.emcdda.europa.eu/publications/ ad-hoc-publication/impact-covid-19-patternsdrug-use-and-harms de.Zugegriffen:05.01.21

38. Chiappini S, Guirguis A, John A, Corkery JM, Schifano $F$ (2020) COVID-19: the hidden impact on mental health and drug addiction. Front Psychiatry 11:767

39. Pogarell O, Wodarz-von Essen $\mathrm{H}$, Wodarz N (2020) SARS-cov-2 Pandemie und Suchterkrankungen: Schwerpunkt Opioidabhängigkeit und Substitution. Sucht 66:271-277

40. Armitage R, Nellums LB (2020) Substance misuse during COVID-19: protecting people who use drugs. Public Health. https://doi.org/10.1016/j. puhe.2020.05.010

41. Dubey MJ, Ghosh R, Chatterjee $S$, Biswas $P$ Chatterjee S, Dubey S (2020) COVID-19 and addiction. Diabetes Metab Syndr 14(5):817-823. https://doi.org/10.1016/j.dsx.2020.06.008

42. Wang QQ, Kaelber DC, Xu R, Volkow ND (2020) COVID-19 risk and outcomes in patients with substance use disorders: analyses from electronic health records in the United States. Mol Psychiatry 14:1-10. https://doi.org/10.1038/s41380-02000880-7

43. Ornell F, Moura HF, Scherer JN, Pechansky F, Kessler FHP, von Diemen L (2020) The COVID19 pandemic and its impact on substance use: implications for prevention and treatment. Psychiatry Res 289:113096. https://doi.org/10. 1016/j.psychres.2020.113096

44. Wei Y, Shah R (2020) Substance use disorder in the COVID-19 pandemic: a systematic review of vulnerabilities and complications. Pharmaceuticals 13(7):155. https://doi.org/10.3390/ph13070155

45. Wittchen HU, Bühringer G, Rehm J (2011) Predictors, Moderators and Outcome of Substitution Treatments - Effekte der langfristigen Substitution Opioid-abhängiger: Prädiktoren, Moderatoren und Outcome (Schlussbericht an das Bundesministerium für Gesundheit.)

46. Uscher-Pines L, Sousa J, Raja P, Mehrotra A, Barnett M, Huskamp HA (2020) Treatment of opioid use disorder during COVID-19: experiences of clinicians transitioning to telemedicine. J Subst Abuse Treat 118:108124. https://doi.org/10.1016/ j.jsat.2020.108124

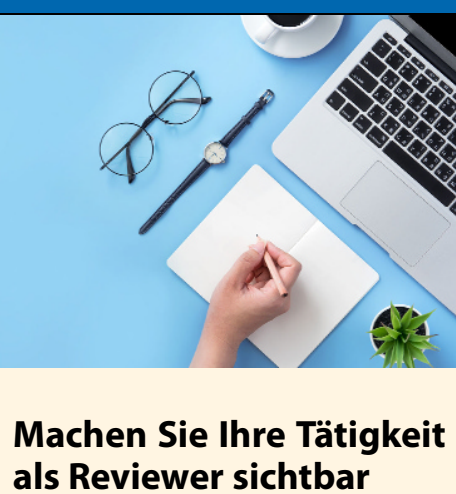

\section{Listen Sie Ihre Gutachten auf publons.com!}

Publons ist eine Onlineplattform, die es Wissenschaftlern ermöglicht, ihre Gutachtertätigkeit bei akademischen Fachzeitschriften sichtbar zu machen. Ziel von Publons ist es, das Peer Review als messbare wissenschaftliche Leistung anzuerkennen. Auf der Seite wird übersichtlich und nachvollziehbar dokumentiert, wie häufig die eigene Expertise in einem speziellen Fachgebiet nachgefragt wurde, was z. B. für Bewerbungsverfahren genutzt werden kann.

Um Ihr Review auf Publons anzugeben, registrieren Sie sich einmalig kostenfrei. Im Anschluss können Sie Ihr Gutachten direkt innerhalb Ihres Profils eingeben. Alternativ leiten Sie die englischsprachige E-Mail, mit der Ihnen der Erhalt des Gutachtens bestätigt wurde, an reviews@publons.com weiter.

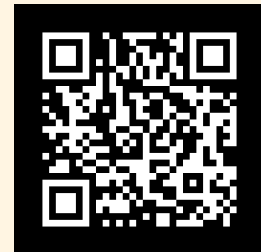

https://publons.com/ 